\title{
Rotating machine prognostics using system-level models
}

\author{
Xiaochuan $\mathrm{Li}^{1}$, Fang Duan ${ }^{1}$, David $\mathrm{Mba}^{1}$, lan Bennett ${ }^{2}$ \\ 1. School of Engineering, \\ London South Bank University, SE1 0AA, London, UK. \\ 2. Shell Global Solutions International, B.V., \\ Rijswijk 2288 GS, Netherlands.
}

\begin{abstract}
The prognostics of rotating machines is crucial for the reliable and safe operation as well as maximizing usage time. Many reliability studies focus on component-level prognostics. However, in many cases, the desired information is the residual life of the system, rather than the lifetimes of its constituent components. This review paper focuses on system-level prognostic techniques that can be applied to rotating machinery. These approaches use multi-dimensional condition monitoring data collected from different parts of the system of interest to predict the remaining useful life at the system level. The working principles, merits and drawbacks as well as field of applications of these techniques are summarized.
\end{abstract}

KEYWORDS: System-level prognostics; rotating machines; condition monitoring; remaining useful life

\section{INTRODUCTION}

Rotating systems such as gas turbines and compressors are widely used due to their high performance and robustness. However, many kinds of failures may occur during the operation of the machine. Those failures will cause unplanned downtime and economic losses as well as reduced reliability. One way to minimize the negative influence of these failures is to make maintenance strategies more predictive by using automated condition monitoring. Condition-based maintenance (CBM) is a preventive maintenance strategy that seeks to improve the reliability of engineering systems based on condition monitoring information [1][2]. CBM enables the diagnosis of impending failures and the prognosis of the future health state and remaining useful life (RUL) of a system [3]. Prognostic programs based on condition monitoring data provide a potent tool for practitioners in making appropriate maintenance decisions by estimating the future degradation trends and anticipating the failure time [4]. Suspensions or overhauls could be carried out before the estimated failure time, which allows for improved machine availability and reliability and reduced the overall operating cost.
Many condition-based reliability studies have focused on component-level prognostics, which enable the failure of critical rotating components to be predicted. However, in many cases, the desired information is the residual life of the system, rather than the lifetimes of its constituent components [5]. This necessitates the development of robust system-level prognostic techniques for rotating machines.

Due to the progress of sensing technology, condition monitoring data such as the oil debris, pressure values, temperature values and vibration is available at different parts of the rotating system [6]. The availability of data from multiple sensors has provided the possibility of developing multidimensional prognostic techniques. Since the RUL of a system is dependent upon its constituent components and how they interact [7], one could make use of the data collected from sensors distributed over the machine and multi-dimensional techniques to predict failure times. Subsequently, system maintenance schedules can be made based on the estimated failure.

Several review papers on prognostic techniques for engineering systems have been published [8] [9] [10] [11] [12] [13]. However, limited numbers of these papers have highlighted the system-level prognostic options for complex rotating machines. This paper reviews prognostic techniques that can be applied to predict rotating machinery failures at the system level.

\section{DISCUSSIONS ON SYSTEM-LEVEL PROGNOSTIC MODELS FOR ROTATING MACHINERY}

\subsection{Characteristics of rotating machinery prognostics}

Compared with general industrial applications, rotating systems have several unique characteristics that should be considered when developing prognostic methods. First, various sources of nonlinearities can be present in complex rotating machines, such as nonlinearities in the bearings, aerodynamic effects and 
friction in rotating assemblies and seals [14]. The presence of these nonlinear elements can lead to nonlinear system dynamic characteristics. Moreover, most real-world rotating machinery works in non-stationary operating conditions, which can have profound effects on both diagnostic and prognostic signals. The non-stationary operations are generated by load/speed variations, system parameter adjustments, strong nonlinearities in the components, etc. $[15,16]$. Moreover, rotating systems are composed of multiple sub-systems and components with various failure modes, which further introduce a degree of complexity in prognostic modelling. In addition to nonlinearity, non-stationary and multiple failure modes, modelers should also consider the possible synergy among the different sensor signals collected from the machine. Therefore, the multidimensional prognostic techniques are commonly used when developing an appropriate systemlevel method for rotating machinery.

\subsection{Prognostic techniques categorization}

The prognostic approaches reviewed in this paper can be divided into three categories: (1) statistical methods, (2) artificial intelligence methods, and (3) similarity-based methods (see Figure 1).

For statistical methods, we review models based on Bayesian theory and proportional hazard models. These models predict the RUL based on past observed information and statistical models in a probabilistic manner. Therefore, a probability density function (PDF) of the RUL is formulated for uncertainty management. Moreover, in statistical methods, machine lifetime data might be required in addition to monitoring data for failure prediction.

When lifetime data is scarce or non-existent, artificial intelligence methods that make predictions using only monitoring data can be considered. These models can be treated as a nonlinear function approximator, which aims to determine dependencies in a training data set such that predictions of the outputs (e.g., RUL) can be made when new inputs are available. Unlike statistical methods, most artificial intelligence methods do not provide a PDF of the RUL. For artificial intelligence methods, we review models based on neural networks and support vector machines (SVMs).

Similarity-based methods are fundamentally different from the techniques in the first two categories because they do not perform trending or extrapolation of the degradation process. Instead, they construct a probabilistic health indicator, which characterizes the system health state via trajectories. Then, predictions are made based on evaluating similarities between the trajectories.

The working principles, merits, drawbacks and the applications of these techniques are discussed in the following sections.

\section{RUL prediction models}

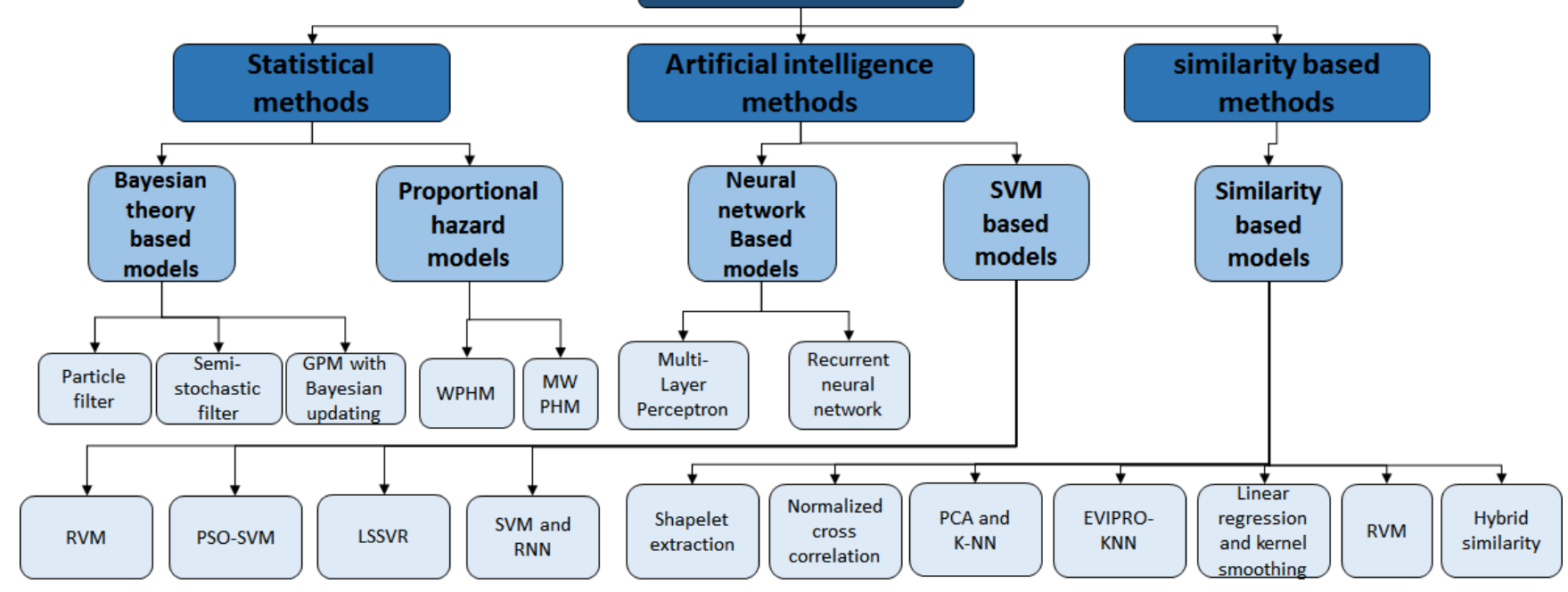

Figure 2 Models categories for RUL prediction

\subsection{Bayesian theory based models}

We assume the system model based on Bayesian theory can be defined as:

$$
\begin{aligned}
& \mathrm{p}\left(x_{t} \mid x_{t-1}\right), \\
& \mathrm{p}\left(y_{t} \mid x_{t}\right)
\end{aligned}
$$

Where $x_{t}$ refers to the unobservable health state of the system under study at time $t, y_{t}$ refers to the observed information at time t. $\mathrm{p}\left(x_{t} \mid x_{t-1}\right)$ is the state equation, and $\mathrm{p}\left(y_{t} \mid x_{t}\right)$ is the observation equation. Then, the prognostic tasks can be divided into two sequential stages: the estimation stage and the prediction stage. The purpose of the estimation stage is to find a state estimate $\mathrm{p}\left(x_{t} \mid Y_{t}\right)$ given the observation history up to time $t$, where $\mathrm{Yt}$ denotes the observation history up to time t. The prediction problem is to predict the health state $\mathrm{p}\left(x_{t+k} \mid Y_{t}\right)$ at a given prediction time $\mathrm{t}+\mathrm{k}$. The RUL can be computed by extrapolating the predicted health state to a pre-set failure threshold [5]. 
Because most engineering facilities in practice undergo a non-linear and time-varying deterioration process, the parameters of the prediction model cannot remain constant over the entire prediction process (otherwise the predictions might be not accurate). Therefore, Bayesian updating methods are often adopted to jointly update the model parameters and the system health state when new observations are available. Three widely used Bayesian updating tools are particle filtering [17] [5], semi-stochastic filtering [18] and dynamic Bayesian updating [19]. To address systems with multiple sensory inputs, sensor fusion techniques, such as principal component analysis [20], independent component analysis (ICA) [21], linear regression [5] and path model [19] are often employed to merge the multivariate measurements into a one-dimensional variable - the system health indicator. Then the equations defined in the first paragraph can be used to map health indicator values to the RUL. Some common Bayesian theory techniques will be discussed below.

a) Particle filtering: The main concept of particle filtering is to represent the required posterior distribution of the state variables, such as $\mathrm{p}\left(x_{t} \mid Y_{t}\right)$, using a set of particles with associated weightings. These particles evolve and adapt recursively when new information becomes available [22]. Then, the unobserved health state is estimated based on these weighted samples.

In health state prediction, particle filter has three advantages: a) it can be applied to nonlinear process with non-Gaussian noise; b) it provides probabilistic results which is helpful to manage the prognostic uncertainties; c) it allows information fusion such that data from multidimensional sensors could be employed collectively [23] [24].

However, one limitation of particle filtering is that a large number of samples might be required to accurately approximate the future state distributions, which may cause the filtering system to collapse. A good solution to this problem is to adopt the efficiency monitoring method of filtering proposed by Carpenter [22].

Wang [17] presented an engine wear estimation model base on particle filtering. In his work, the relationship between condition monitoring measurements and system wear was modelled using the concept of a floating scale parameter. In this approach, the scale parameter of the observations is a function of both system degradation and time. The PCA was employed to produce a one-dimension representation of the metal concentration data, which were then processed by particle filtering to obtain the density function of system wear. Recently, Sun et al. [5] applied a state space model embedded with particle filtering to a gas turbine degradation data set obtained via simulation. A health indicator inferred using a linear regression method was used to represent the latent degradation state of the engine given multivariate sensory measurements. The authors combined the state estimation with model parameter estimation to reduce the prognostic uncertainty. b) Semi-stochastic filtering: Wang and Christer [25] firstly developed a state space prognostic model embedded with a semi-stochastic filtering technique. Based on the authors assumption, two relationships should be determined to model the probability density of a system's state given all of the observations: the relationship between $x_{t}$ and $x_{t-i}$ and the relationship between $y_{t}$ and $x_{t} . x_{t}$ is the residual life at time $\mathrm{t}$, and $y_{t}$ denotes the observation at time t. The two relationships could be described by $x_{t}=x_{t-i}-(t-i)$ and $y_{t}=$ $g\left(x_{t}, \delta_{t}\right)$, where $\delta_{t}$ denotes a noise term and $g$ is a function to be determined. Then, the posterior distribution of the residual life given all past observation history can be estimated based on the obtained conditional probabilities. Various extensions have been developed and applied to rotating system prognostics based on the above framework. A revision of this semi-stochastic filtering technique was applied to the lifetime data and monitored oil analysis data collected from an aircraft engine [20]. The predicted RUL is assumed to be proportional to the wear increment measured by the monitored measurements. PCA was employed to obtain a weighted average of the original monitored data. A similar model was given in [26]. The authors combined lifetime data and accumulative metal concentration data to estimate the remaining useful life of a diesel engine. Similarly, Wang and Hussin [21] developed a stochastic filtering-based prognostic model and applied it to two data sets: engine lubricant and contaminant analysis data and metal concentration data. Instead of the commonly used PCA, they employed ICA to merge the latter. The results indicated that a higher accuracy was achieved based on the data lubricant and contaminant sets. Another extension of Wang's semi-stochastic filtering was given in [27]. This model extends the original filtering with respect to two aspects: the concept of a two-stage life model was introduced to achieve both fault detection and prediction, and a combination of categorical and continuous hidden Markov chain was used to model the underlying health state transitions. The authors suggested the use of a PCA algorithm in combination with the proposed model to address multidimensional data in complex rotating systems.

c) General path model with dynamic Bayesian updating: Coble and Hines [19] developed a prognostic model, called the general path model (GPM), to predict the RUL of aircraft engines. First a deterioration measure is identified to represent the failure evolution. Then a linear regression fit of the measure is extrapolated to a preset failure threshold to predict the RUL. The results indicated that the dynamic Bayesian updating method greatly improved the prediction accuracy.

These approaches are based on the same assumption that there are no maintenance actions during two condition check points or that the actions do not affect the system degradation pattern. However, this may not be the case in reality. Moreover, failure lifetime data may be required for parameter estimation for most models 
mentioned above. But this type of data might be scarce in reality.

\subsection{Proportional Hazard Models}

Machine failures can be predicted by analyzing either condition monitoring data or historical service lifetime data [28] [29]. Condition monitoring data, which are obtained continuously, have been widely used to predict fault evolutions and system RUL. The lifetime data, which indicate how long the machine has been operating since the last failure (or suspension), also provide supplementary information for RUL prediction [30]. Hence, it would be wise to develop proper prognostic models with a combination of condition monitoring data and lifetime data. The proportional hazard model (PHM), proposed by Cox [31], attempts to utilize both types of information for RUL prediction. The basic assumption of this method is that the failure rate of a machine depends on two factors: the baseline hazard rate and the effects of covariates (condition monitoring sensory variables). Hence, the hazard rate of a system at service time $t$ can be written as $\lambda(\mathrm{t} ; \mathrm{z})=\lambda_{0}(t) \exp (z \beta)$, where $\lambda_{0}(t)$ denotes the base line hazard, which is determined by the system lifetime data. $\exp (z \beta)$ denotes the covariate function, which describes the effect of the sensory variables on the degradation process [28]. Applying PHMs requires that the baseline hazard function $\lambda_{0}(t)$ and covariate function $\exp (z \beta)$ be identified.

Methods that have been used to estimate the $\exp (z \beta)$ including the maximum likelihood algorithm [31] [4] and Wald statistic [30]. The relative influences of sensory signals on the system hazard rate are first determined. Then, key variables with close correlation to the system failure are retained and employed to estimate the system failure probability density [32]. Once the covariate function has been determined, the baseline function parameters can be estimated. The PHM provides a distribution-free estimate of the baseline function $\lambda_{0}(t)$ [32], which means that a specific distribution for $\lambda_{0}(t)$ is not needed to fit the lifetime data. Researchers prefer this type of estimate because it can avoid the loss of accuracy caused by the assumption of a parametric distribution [33]. However, in practice, the baseline hazard function is often assumed to be a parametric distribution, such as the Weibull or exponential distribution [12]. Such assumptions might not be reasonable in many cases because of the confusing effects of different covariates [32].

To apply the PHMs, the sensory measurements and lifetime data are combined to fit the model. The PHM can identify the important risk factors from all input variables and their relative influence on the failure of the equipment [30]. Then, the system failure distribution at time service $t$ can be estimated. Finally, the failure time at time t can be predicted according to the estimated probability distribution.

PHMs have been applied to many non-linear and non-stationary machinery prognostic problems. Jardine et al. [34] developed a PHM and employed it to estimate the remaining useful life of aircraft engines and marine gas turbines. The baseline hazard function was assumed to be a Weibull distribution and was estimated using lifetime data. The levels of various metal particles (such as $\mathrm{Fe}, \mathrm{Cu}$ and $\mathrm{Mg}$ ) in the oil were used as the covariates in both cases. The influence of the condition monitoring variables on the equipment RUL has been properly interpreted by the developed PHM. The authors also applied the PHM to estimate the RUL and optimize the maintenance decisions of haul truck wheel motors in [30]. The key covariates related to failures were identified from 21 monitored oil analysis variables using the developed PHM. The results showed that significant savings in maintenance costs could be achieved by optimizing the overhaul time as a function of lifetime data and oil analysis variables.

The above models are based on the assumption that the system under study is subject to a single failure mode. However, in practice, most complex mechanical systems consist of multiple sub-systems with various failure modes [28]. Therefore, prognostic models for determining only one type of failure mode cannot properly estimate the overall system failure time. Recently, Zhang et al. [28] proposed a mixed Weibull proportional hazard model (MWPHM) for complex mechanical system reliability assessment. In this model, the overall system failure probability density is determined by a mixture of failure densities of various failure modes. The influences of multiple monitoring signals on different failure modes are integrated using the maximum likelihood estimation algorithm. Real data from a centrifugal water pump were combined with lifetime data to test the robustness of the model.

The main problem with applying PHMs for failure prediction is that they require a large amount of lifetime data to determine the parameters of the baseline hazard function and the weighting of covariates [29], which may limit the applications of PHMs because the amount of lifetime data might be insufficient in many cases due to various reasons, such as missing or no records or transcription mistakes [35]. Another drawback of PHMs is that they rely on the choice of the failure threshold for RUL prediction. The threshold must be continuously updated when system maintenance is conducted [28].

\subsection{Neural network based models}

Artificial neural networks (ANNs) have recently been widely used in modelling degradation processes. An ANN is a computing system that is able to capture, represent and compute mapping from the input multivariable space to the output space [36]. ANNs have three layers: an input layer, one or more hidden layers and an output layer. ANNs are comprised of a large number of processing elements (known as neurons) that are connected to each other by weighted interconnections [37]. These neurons are organized into distinct layers, and their interconnections are determined through a training process. The network training involves presenting data 
sets collected from the degradation process. Then the network parameters are adjusted to minimize the errors between the model output and desired output [36]. Once the training is finished, ANNs process new input data to make predictions about the outputs (RUL).

Network architectures that have been used for prognostics can be classified into two types: feed-forward and recurrent networks [38]. In feed-forward networks, the signals flow in one direction; therefore, the inputs to each layer depend only on the outputs of the previous layer. However, applications in signal processing and prognostics should consider the system dynamics. Recurrent networks provide an explicit dynamic representation by allowing for local feedbacks [39]. Two types of networks: multi-layer perceptron (MLP) and recurrent neural networks (RNNs) (Figure 2 shows the architecture of a simple RNN), which have been applied extensively by researchers, will be discussed below [36] [40].

a) Multi-Layer Perceptron (MLP): MLPs are one of the most popular feed-forward neural networks used for prognosis [12]. MLPs utilize back-propagation (BP) learning technology for training. After the training, the MLP is capable of classifying the fault and predicting the RUL based on new measurements collected from machines [41]. The benefit of back-propagation (BP) training is that it does not require knowledge of the precise form of the input-output mapping functions (e.g., function type, number of model parameters) of the model to be built, which makes it suitable for the analysis of multivariate complex systems [40].

b) Recurrent neural network (RNN): Feed-forward neural networks have limitations in identifying temporal dependences in time series signals [42]. RNNs solve this problem by including local or global feedback between neurons. Thus, they are suitable for a wide range of dynamic systems [42], such as time-varying and nonlinear systems. However, the drawback of RNNs is the limitations in accurate long-term predictions arising from the frequently used gradient descent training algorithm [42].

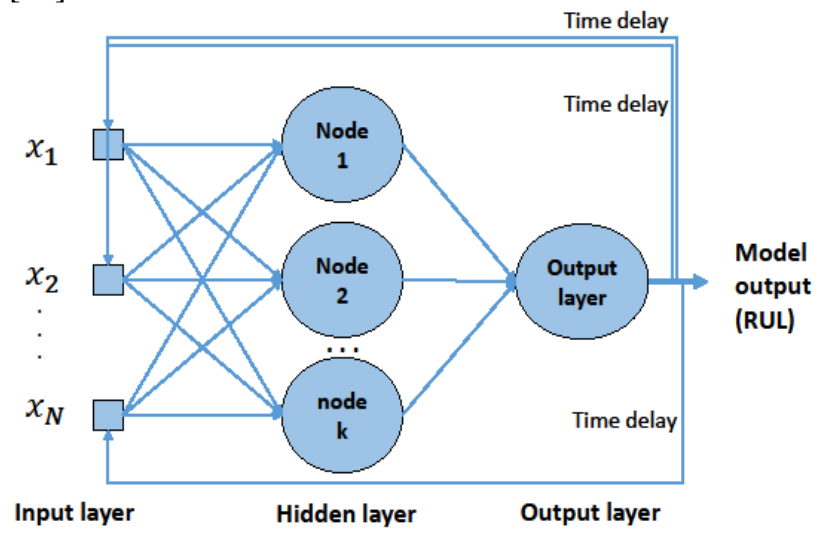

Figure 2 Architecture of a simple RNN

ANNs can represent and build mappings from experience and history measurements to predict the RUL and then adapt it to unobserved situations. The strong learning and generalization capabilities of ANNs render them suitable for modelling complex processes [37], particularly systems with nonlinear and time-varying dynamics [41] [43] - [45]. ANNs are superior in capturing and depicting relationships between many variables in high-dimensional data space [40] [45] [46] [37]. RNNs are suitable for approximating dynamic dependencies [42]. These distinct characteristics make ANNs promising candidates for modelling degradation processes in rotating machinery.

$\mathrm{Xu}$ et al. [47] successfully employed RNNs, support vector machines (SVMs) and Dempster-Shafer regression to estimate the RUL of an aircraft gas turbine. Echo state network (ESN), which is a variant of the RNNs, was employed by Peng et al. [48] to predict the RUL of engines using NASA repository data. The results indicated that the ESN significantly reduced the computing load of the traditional RNNs. ANNs have also been used in combination with Kalman filters and Extended Kalman filters in [49] and [50] to perform failure predictions of aircraft engines.

Although ANNs have been shown the superior power in addressing complex prognostic problems which have multivariate inputs, there are some limitations. For example, the majority of the ANN prognostic models aim to assume a single failure mode and do not relate lifetime data with the machine RUL. Moreover, the models rely on a large amount of data for training. The prognostic accuracy is closely dependent on the quality of the training data [47]. Furthermore, ANNs allow for few explanatory insights into how the decisions are reached (also known as the black box problem), which has become a concern to modelers because the causal relationship between the model variables is essential for explaining the fault evolution [51]. Attempts to solve the black box problem can be found in [52]. Moreover, ANNs lack a systematic approach to determine the optimal structure and parameters of the network to be established [40].

\subsection{Support vector machine based models}

Previously, SVMs were mainly used for pattern recognition problems and have not been used for time series forecasting until the introduction of the Vapniks insensitive loss function [9]. SVM-based machine learning starts with a number of input variables $\mathrm{x}(\mathrm{i}), \mathrm{i}=$ $1,2,3, \ldots, \mathrm{N}$, and the corresponding target values $\mathrm{y}(\mathrm{i}), \mathrm{i}=$ $1,2,3, \ldots, N$. The idea is to learn the dependency of $y(i)$ on $\mathrm{x}(\mathrm{i})$ and to define a function over the input variables. Then, predictions of $y(i)$ can be made given unseen $\mathrm{x}(\mathrm{i})$ [53]. When applying SVMs to nonlinear prognostics, model inputs are first mapped onto a higher dimensional feature space by using a kernel function. The most commonly employed kernel function is the radial-based function (RBF) [54]. Then a linear model is constructed in the feature space to make estimation. Figure 3 shows the architecture of a simple SVM based prognostic model. SVMs are excellent in addressing prognostic problems 
regarding complex rotating machinery because they have no limitations on the dimensionality of the input vectors and have relatively a low computational burden [13]. Besides, SVMs can achieve highly accurate results with nonlinear inputs [54].

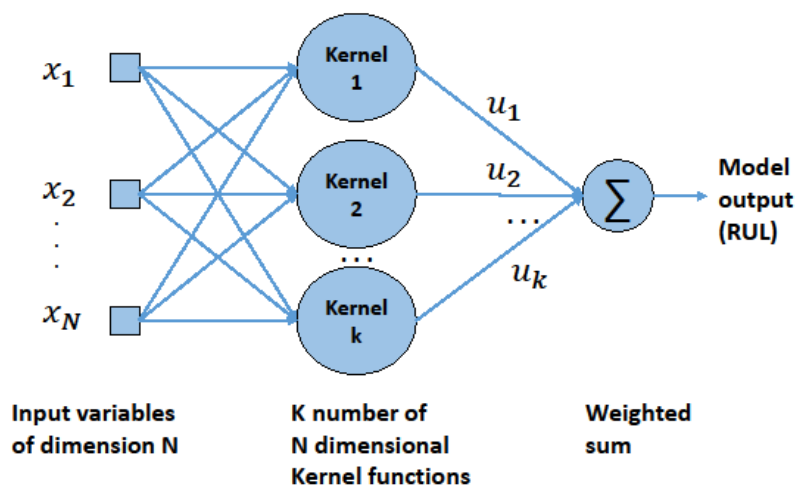

Figure 3 Architecture of a simple SVM based prognostic model

Several different prognostic models based on SVMs have been used in modelling nonlinear and non-stationary dynamic systems.

a) Relevance vector machine (RVM): RVM has an identical function form as a SVM. Hu and Tse [55] proposed a model based on RVM for RUL prediction of a pump. This model was proved to be accurate when dealing with non-stationary vibration signals.

b) Particle swarm optimization and SVM (PSOSVM): Garcia Nieto et al. [56] developed an RUL framework based on the PSO-RBF-SVM technique. This model combines a SVM with particle swarm optimization (PSO) to enable the parameter adjustment of the RBF kernel function. The results show that the proposed prognostic model accurately predicts the RUL of engines based on a simulation data set (collected from the MAPSS).

c) Least squares support vector regression and hidden Markov model (LSSVR and HMM): Compared with the traditional SVM, LSSVR can lead to better performance in addressing non-linear, small sample problems. Li et al. [57] proposed a hybrid model of the LSSVR and the HMM for RUL prediction. The RUL was calculated by the LSSVR model built on the health indexes obtained from HMM.

d) SVM and RNN: In [47], a SVM approach based on the RBF kernel function was employed together with a RNN and Dempster-Shafer regression to predict the RUL of engines. The integrated prognostic method demonstrated superior capacity in providing accurate predictions.

However, the problem with using SVM is that a standard method of choosing an appropriate kernel function for SVMs does not exist [9]. In addition, parameters should be specifically tuned for the case of interest and this might be challenging. Efforts should be made to choose the appropriate kernel functions and estimate the appropriate parameters.

\subsection{Similarity based models}

Similarity-based prognostic models are particular cases of data-driven models and have only recently been applied to complex rotating machinery. These models are essentially pattern matching approaches [58]. Similaritybased prognostic models are suitable for situations in which abundant run-to-failure data of a mechanical system are available [59]. Multivariate monitoring data collected from various failure modes and operating conditions [58] of the system are first processed to produce a health indicator (HI). The indicator represents the fault evolution of the system by trajectories. The methods to obtain the health indicator trajectories include logistic regression [59], weighted averaging methods [60], and flux-based methods [61]. If the un-processed data already capture the progression of the degradation process, the data can remain multi-dimensional [62]. Then, the monitoring data are converted into instances. An instance can be either a segment of the HI trajectories or a complete degradation trajectory. Therefore, a library of instances can be created from these run-to-failure data and then stored in the memory. If one wishes to predict the RUL using a new run-to-failure dataset, the same operations are applied to the new data to produce a new instance. Instead of extrapolating, the instance is compared with the stored instances to determine and select the instances with the best matching scores (i.e., the most similar ones) [59]. Then, the best matching instance is used to extrapolate the RUL or the weighted multiple instances are added together to calculate the RUL [58].

Figure 4 shows the general framework of similaritybased prognostic models. Because the similarity-based approaches use training data to construct instances (health indicator trajectories or multidimensional monitoring variables), they are compatible with algorithms that extract health indicators for RUL prediction [62]. The advantage of similarity-based approaches is that they can achieve satisfactory and accurate predictions when abundant data are collected from a variety of failure modes. However, the run-to-failure data are scarce in many cases [58]. Hence, efforts should be made to extend this type of approach to situations in which limited training data are available. Additionally, many similaritybased prognostic techniques suffer from computational inefficiency in terms of sorting a large amount of training data [63].

The ability to accommodate multidimensional sensory measurements collected from various failure patterns makes similarity-based methods suitable for the prognostics of complex rotating machinery. Examples are given below to set out how various similarity based models have been used for RUL prediction. 


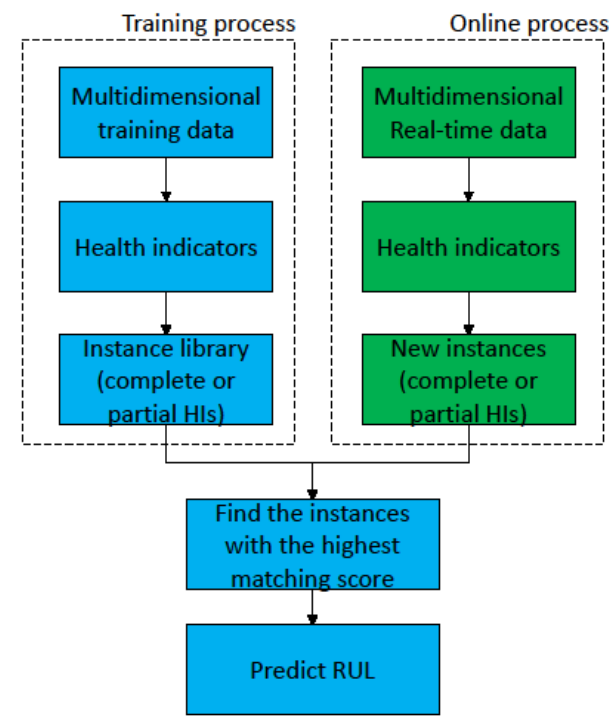

Figure 4 General framework of similarity-based prognostic models

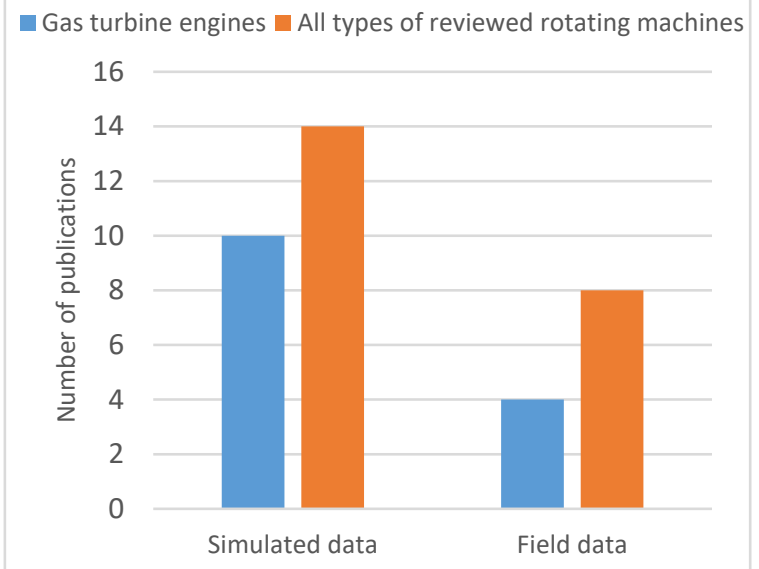

Figure 5 Type of data used in studies regarding (a) Gas turbine engines only (blue), and (b) All kinds of reviewed rotating machines (orange)

a) Similarity model based on shapelet extraction: Malinowski et al. [62] developed an RUL prediction technique that employs the Shapelet extraction process to extract failure patterns from multivariate sensory data obtained from a turbofan engine simulation program, CMAPSS. The RUL was calculated as the weighted sum of the failure patterns that are highly corrected with the residual life. b) Similarity model based on normalized cross correlation: Zhang et al. [64] applied a prognostic method based on the similarity of phase space trajectory to the monitoring data collected from a pump with six distinct degradation modes. The normalized cross correlation was employed to determine the optimal matching trajectory segments, which were then used to estimate the RUL.

c) Similarity model based on PCA and K-NN: Mosallam et al. [65] employed principle component analysis (PCA) and an empirical mode decomposition (EMD) algorithm to construct health indicators from turbofan engine deterioration simulation data. Then, the knearest neighbor (K-NN) classifiers were used to determine the most similar HIs for RUL prediction.

d) Similarity model based on belief functions: An improved technique based on belief functions was proposed by Ramasso and Gouriveau [63] and Ramasso [66]. In this method, the authors only match the last points of the trajectories in the library with tested ones because the last points are more likely to be closely related to the degradation state. One of the main contributions of this method is its ability to manage labels (indicating degradation states) that would have been incorrectly assigned to the sensory data.

e) Similarity model based on linear regression and kernel smoothing: Wang et al. [59] proposed a prognostic model in which the health indicator is constructed from multiple sensors using linear regression. The best matching instances were selected by examining the Euclidean distance between the test and stored instances. This method was applied to data provided by the 2008 PHM Data Challenge Competition to predict the RUL of an unspecified system.

f) Similarity model based on regression vector machine (RVM): Wang et al. [67] improved the previous model by incorporating the uncertainty information into the RUL estimation. The degradation curves of health indexes were estimated using RVM. The Challenge data was employed again to test the effectiveness of this method.

g) Hybrid similarity model: Hu et al. [68] proposed an ensemble prognostic model which combines five individual algorithms (i.e. similarity-based approach with the SVM, RVM and Exponential fitting, a Bayesian linear regression with the quadratic fitting and a RNN) with a weighted sum formulation. The integrated model shows higher accuracy in RUL prediction compared to any single algorithm. 
Table 1 Applications of RUL prediction models

\begin{tabular}{|c|c|c|}
\hline $\begin{array}{l}\text { Rotating machine } \\
\text { type }\end{array}$ & RUL prediction models & Common available data types \\
\hline Gas turbine engines & $\begin{array}{l}\text { Similarity model based on shapelet extraction (Malinowski et al. [62]) } \\
\text { Similarity model based on linear regression and kernel smoothing (Wang et al. [59]) } \\
\text { Similarity model based on PCA and K-NN (Mosallam et al. [65]) } \\
\text { Similarity model based on belief functions (Ramasso et al. [66]) } \\
\text { Similarity model based on RVM (Wang et al. [67]) } \\
\text { Hybrid similarity model based on SVM, RVM, Exponential fitting, Quadratic fitting and } \\
\text { RNN (Hu et al. [68]) } \\
\text { Echo state network (Peng et al. [48]) } \\
\text { Multi-layer perceptron and kalman filter (Peel et al. [49]) } \\
\text { Recurrent neural network and extended kalman filter (Heimes [50]) } \\
\text { Recurrent neural network and support vector machine (Xu et al. [47]) } \\
\text { Particle filtering and linear regression (Wang [17], Sun et al. [5]) } \\
\text { Semi-stochastic filtering and PCA (Wang [20], Wang and Hussin [21]) } \\
\text { Linear regression and dynamic Bayesian updating (Coble and Hines [19]) } \\
\text { Weibull proportional hazard model (Jardine et al. [34]) } \\
\text { PSO-SVM (Garcia Nieto et al. [56]) } \\
\text { Least squares support vector regression and HMM (Li et al. [57]) }\end{array}$ & $\begin{array}{l}\text { 1. Condition monitoring data: } \\
\text { Vibration, } \\
\text { metal concentration, acoustic } \\
\text { emission, ratio of fuel flow, } \\
\text { temperature of Fan, low/high } \\
\text { pressure compressor and low/high } \\
\text { pressure turbine, } \\
\text { pressure, } \\
\text { fan speed, etc. } \\
\text { 2. Lifetime data }\end{array}$ \\
\hline Pumps & $\begin{array}{l}\text { Similarity model based on normalized cross correlation (Zhang et al. [64]) } \\
\text { Mixture of Weibull proportional hazard model (Zhang et al. [28]) } \\
\text { Relevance vector machine (Hu and Tse [55]) }\end{array}$ & $\begin{array}{l}\text { 1. Condition monitoring data: } \\
\text { Mainly vibration, can involve } \\
\text { pressure, temperature, etc. } \\
\text { 2. Lifetime data }\end{array}$ \\
\hline Diesel engines & Semi-stochastic filtering and PCA (Wang [26]) & $\begin{array}{l}\text { 1. Condition monitoring data: } \\
\text { Metal concentration, etc. } \\
\text { 2. Lifetime data }\end{array}$ \\
\hline $\begin{array}{l}\text { Haul truck wheel } \\
\text { motors }\end{array}$ & Weibull proportional hazard model (Jardine et al. [30]) & $\begin{array}{l}\text { 1. Condition monitoring data: } \\
\text { sediment, viscosity, voltage, load, } \\
\text { vibration, etc. } \\
\text { 2. Lifetime data }\end{array}$ \\
\hline
\end{tabular}

\section{SUMMARIES OF PROGNOSTIC TECHNIQUES OF ROTATING MACHINES}

Table 1 summarizes the application of different RUL prediction models to various industrial rotating machines and the machines' common available data types.

Furthermore, the reviewed manuscripts (those in Table1) are classified based on the type of data used in the article. There are two types of data, namely, simulated data collected from simulation programs, such as C-MAPSS, and field data (real-world condition monitoring data). Fig.5 compares the type of data being used in studies regarding a) gas turbine engines only and (b) all types of reviewed rotating machines (including gas turbine engines, diesel engines, haul truck wheel motors and pumps).

According to the reviewed articles, the RUL estimate of gas turbine engines is the main application field. However, the proportion of studies using simulation data is higher than those using field data. The reason for this is the simplicity of using simulation programs and the difficulty of obtaining sufficient field data from operating machines.

\section{DISCUSSIONS ON RELIABILITY ANALYSIS OF SYSTEM WITH MULTIPLE FAILURE MODES}

Most existing prognostic techniques were originally developed for a single failure mode. To predict RUL for systems with multiple failure modes, several models must be separately constructed for each failure mode. For example, Daigle et al. [69] developed a distributed method for failure prediction of a four-wheel rover. This method first decomposes the system-level prognostic problem into independent sub-failure problems through structural model decomposition. Thereafter, the Kalman filter and physical model are used to perform individual failure prognostics. Finally, the local prognostic results are merged to form a system-level result. However, the correlation between different failures may be overlooked via this approach. To solve this problem, several frameworks for reliability analysis in general engineering systems with competitive multiple failures have been proposed. Ahmad et al. [70] developed a failure analysis approach by integrating the failure mode effect and criticality analysis (FMECA) and PHM. This method was validated in a cutting process system with two failure modes. FMECA was applied to classify the censored and 
uncensored data based on the severity of the different failure modes. The FMECA output was then used in PHM to determine the reliability of the system. Huang and Askin [71] proposed a method to analyze the reliability of an electronic device with two types of competing failure modes. Based on the competing failure rule, the mean time-to-failure of the device was estimated by jointly considering the failure rate of both failure modes. Bichon et al. [72] proposed a surrogate-based approach based on the Gaussian process model and physical laws. This method was used to analyze the failure probability of a liquid hydrogen tank with three failure modes. However, most of the frameworks discussed above have not yet been used to predict the RUL for rotating machinery. Therefore, efforts can be made to extend them to rotating machine prognostics.

\section{CONCLUSIONS}

This paper has explored prognostic models for predicting the remaining useful life of rotating machines at the system level. The reviewed prognostic models make predictions based on the multi-dimensional condition monitoring signals collected from the sensors distributed over the studied system. The relevant theories were discussed, and the advantages and disadvantages of the main prognostic model classes were explored. Examples were given to explain how these approaches have been applied to predict the RUL of rotating systems. The reviewed approaches generally require a large amount of historical data (condition monitoring data or lifetime data) to obtain accurate estimates. In addition, the implementation of the reviewed models in industry is still in the nascent stage and more work should be conducted to apply them in real-world operating machines. Moreover, most of the reviewed techniques were originally designed for a signal failure mode. Therefore. several frameworks for reliability analysis of general engineering systems with multiple failure modes were examined. In the future, more work should be conducted to apply these frameworks to rotating machinery prognostics.

\section{REFERENCES}

[1] J. Veldman, W. Klingenberg, and H. Wortmann. Managing condition-based maintenance technology. Journal of Quality in Maintenance Engineering, 17(1):40-62, 2011.

[2] D. A. Tobon-Mejia, K. Medjaher, and N. Zerhouni. CNC machine tools wear diagnostic and prognostic by using dynamic Bayesian networks. Mechanical Systems and Signal Processing, 28:167-182, 2012.
[3] Y. Peng, M. Dong, and M.J. Zuo. Current status of machine prognostics in condition-based maintenance: a review. The International Journal of Advanced Manufacturing Technology, 50(14):297-313, 2010.

[4] V.T. Tran, H.T. Pham, B.S. Yang, and T.T. Nguyen. Machine performance degradation assessment and remaining useful life prediction using proportional hazard model and support vector machine. Mechanical Systems and Signal Processing, 32:320-330, 2012.

[5] J. Sun, H. Zuo, W. Wang, and M.G. Pecht. Application of a state space modeling technique to system prognostics based on a health index for condition-based maintenance. Mechanical Systems and Signal Processing, 28:585-596, 2012.

[6] W. Wang and A.H. Christer. Towards a general condition based maintenance model for a stochastic dynamic system. Journal of the Operational Research Society, 51(2):145-155, 2000.

[7] M. Daigle, A. Bregon, and I. Roychoudhury. A Distributed Approach to System-Level Prognostics. In Annual conference of the Prognostics and Health management Society 2012, pages 71-82, 2012.

[8] A. Heng, S. Zhang, A.C. Tan, and J. Mathew. Rotating machinery prognostics: State of the art, challenges and opportunities. Mechanical Systems and Signal Processing, 23(3):724-739, 2009.

[9] M.S. Kan, A.C. Tan, and J. Mathew. A review on prognostic techniques for non-stationary and non-linear rotating systems. Mechanical Systems and Signal Processing, 62:1-20, 2015.

[10] J. Lee, F. Wu, W. Zhao, M. Ghaffari, L. Liao, and D. Siegel. Prognostics and health management design for rotar machinery systems - Reviews, methodology and applications.

[11] X. Si, W.Wang, C. Hu, and D. Zhou. Remaining useful life estimation A review on the statistical data driven approaches. European Journal of Operational Research, (1):1-14.

[12] J.Z. Sikorska, M. Hodkiewicz, and L. Ma. Prognostic modelling options for remaining useful life estimation by industry. Mechanical Systems and Signal Processing, 25(5):18031836, 2011.

[13] L. Zhang, Z. Liu, D. Luo, J. Li, and H. Huang. Review of Remaining Useful Life Prediction Using Support Vector Machine for Engineering Assets. 2013 International Conference on Quality, Reliability, Risk, Maintenance, and Safety Engineering (QR2MSE), pages 17931799, 2013. 
[14] S. T. Noah and P. Sundararajan. Significance of Considering Nonlinear Effects in Predicting the Dynamic Behavior of Rotating Machinery. Journal of Vibration and Control, 1(4):431-458, 1995.

[15] A. Martini, M. Troncossi, A. Rivola, and D. Nascetti. Advances in Condition Monitoring of Machinery in Non-Stationary Operations. Lecture Notes in Mechanical Engineering, 5:535-544, 2014.

[16] W. Bartelmus, F. Chaari, R. Zimroz, and M. Haddar. Modelling of gearbox dynamics under time-varying nonstationary load for distributed fault detection and diagnosis. European Journal of Mechanics, A/Solids, 29(4):637-646, 2010.

[17] W. Wang. A prognosis model for wear prediction based on oil-based monitoring. Journal of Operational Research Society, 58(7):887-893, 2007.

[18] W. Wang. A model to determine the optimal critical level and the monitoring intervals in condition-based maintenance. International Journal of Production Research, 38(6):14251436, 2000.

[19] J. Coble and J. Hines. Applying the General Path Model to Estimation of Remaining Useful Life. International Journal of Prognostics and Health Management, 2(1):1-13, 2011.

[20] W. Wang and W. Zhang. A model to predict the residual life of aircraft engines based upon oil analysis data. Naval Research Logistics, 52(3):276-284, 2005.

[21] W. Wang and B. Hussin. Plant Residual Time Modelling Based on Observed Variables in Oil Samples. The Journal of the Operational Research Society, 60(6):789-796, 2009.

[22] J. Carpenter, P. Clifford, and P. Fearnhead. Improved particle filter for nonlinear problems. In IEE Proceedings - Radar, Sonar and Navigation, volume 146, page 2, 1999.

[23] M. Orchard, B.Q. Wu, and G. Vachtsevanos. A particle filtering framework for failure prognosis. In Proceedings of WTC2005 World Tribology Congress III, pages 1-2, Washington, D.C., 2005.

[24] C.C. Chen, B. Zhang, and G. Vachtsevanos. Prediction of machine health condition using neuro-fuzzy and bayesian algorithms. IEEE Transactions on Instrumentation and Measurement, 61(2):297- 306, 2012.

[25] W. Wang and A.H. Christer. Towards a general condition based maintenance model for a stochastic dynamic system. J Oper Res Soc, 51(2):145-155, 2000.

[26] W. Wang, B. Hussin, and T. Jefferis. A case study of condition based maintenance modelling based upon the oil analysis data of marine diesel engines using stochastic filtering. International Journal of Production Economics, 136(1):84-92, 2012.

[27] W. Wang. A two-stage prognosis model in condition based maintenance. European Journal of Operational Research, 182(3):1177-1187, 2007.

[28] Q. Zhang, C. Hua, and G. Xu. A mixture Weibull proportional hazard model for mechanical system failure prediction utilising lifetime and monitoring data. Mechanical Systems and Signal Processing, 43(1-2):103112, 2014.

[29] Y. Sun, L. Ma, J. Mathew, W. Wang, and S. Zhang. Mechanical systems hazard estimation using condition monitoring. Mechanical Systems and Signal Processing, 20(5):1189-1201, 2006.

[30] A.K.S. Jardine, D. Banjevic, M. Wiseman, S. Buck, and T. Joseph. Optimizing a mine haul truck wheel motors condition monitoring program Use of proportional hazards modeling. Journal of Quality in Maintenance Engineering, 7(4):286-302, 2001.

[31] B.Y. and D.R. Cox. Models and Life-Tables Regression. Journal of the Royal Statistical Society, 34(2):187-220, 1972.

[32] A. Bendell. Proportional Hazards Modelling in Reliability Assessment. Reliability Engineering, 11:175-183, 1985.

[33] Z. Li, S. Zhou, S. Choubey, and C. Sievenpiper. Failure event prediction using the Cox proportional hazard model driven by frequent failure signatures. IIE Transactions, 39(3):303315, 2007.

[34] A. K. S. Jardine, P. M. Anderson, and D. S. Mann. Application of the Weibull Proportional Hazards Model To Aircraft and Marine Engine Failure Data. Quality and Reliabaility Engineering International, (October):77-82, 1986.

[35] C. Manuel, S. Carlos, M. Pereira, C. Joao, and C.D. Oliveira. Data management for CBM optimization. Journal of Quality in Maintenance Engineering, 12(1):37-51, 2008.

[36] M. Y. Rafiq, G. Bugmann, and D. J. Easterbrook. Neural network design for engineering applications. Computers and Structures, 79(17):1541- 1552, 2001.

[37] J. A. Rodriguez, Y. El Hamzaoui, J. A. Hernandez, J. C. Garcia, J. E. Flores, and A. L. Tejeda. The use of artificial neural network (ANN) for modeling the useful life of the failure assessment in blades of steam turbines. Engineering Failure Analysis, 35:562-575, 2013. 
[38] A.F. Atiya, S.M. El-Shoura, S.I. Shaheen, and M.S. El-Sherif. A comparison between neuralnetwork forecasting techniques - Case study: River flow forecasting. IEEE Transactions on Neural Networks, 10(2):402-409, 1999.

[39] T. Liu and R. Gencay. Nonlinear modelling and prediction with feedforward and recurrent networks. Physica D, 108:119-134, 1997.

[40] Z. Zhang, Y. Wang, and K. Wang. Fault diagnosis and prognosis using wavelet packet decomposition, Fourier transform and artificial neural network, 2012.

[41] F. Ahmadzadeh and J. Lundberg. Remaining useful life prediction of grinding mill liners using an artificial neural network. Minerals Engineering, 53:1-8, 2013.

[42] J. Liu, D. Djurdjanovic, J. Ni, N. Casoetto, and J. Lee. Similarity based method for manufacturing process performance prediction and diagnosis. Computers in Industry, 58(6):558-566, 2007.

[43] T. Senjyu, H. Takara, K. Uezato, and T. Funabashi. One-hour-ahead load forecasting using neural network. Power Systems, IEEE, 17(1):113-118, 2002.

[44] J. Connor, R. Martin, and L. Atlas. Recurrent Neural Networks and Robust Time Series Prediction. Neural Networks, IEEE, 5(2):240 254, 1994.

[45] S. Zhang and R. Ganesan. Multivariable trend analysis using neural networks for intelligent diagnostics of rotating machinery. Journal of Engineering for Gas Turbines and Power, 119(2):378, 1997.

[46] S. Wang. Application of self-organising maps for data mining with incomplete data sets. Neural Computing and Applications, 12(1):42-48, 2003.

[47] J. Xu, Y. Wang, and L. Xu. PHM-oriented integrated fusion prognostics for aircraft engines based on sensor data. IEEE Sensors Journal, 14(4):1124-1132, 2014.

[48] Y. Peng, H. Wang, J. Wang, D. Liu, and X. Peng. A Modified Echo State Network based Remaining Useful Life Estimation Approach. Prognostics and Health Management (PHM), 2012 IEEE Conference on, pages 1-7, 2012.

[49] L. Peel and I. Gold. Data Driven Prognostics using a Kalman Filter Ensemble of Neural Network Models. International Conference on Prognostics and Health Management 2008, pages 1-6, 2008.

[50] F.O. Heimes. Recurrent Neural Networks for Remaining Useful Life Estimation. Prognostics and Health Management, 2008. PHM 2008. International Conference on, pages 1-6, 2008.
[51] J.D. Olden and D.A. Jackson. Illuminating the black box: a randomization approach for understanding variable contributions in artificial neural networks. Ecological Modelling, 154(12):135-150, 2002. 9

[52] D. Sussillo and O.Barak. Opening the black box: low-dimensional dynamics in high-dimensional recurrent neural networks., 2013.

[53] B. Saha, K. Goebel, and J. Christophersen. Comparison of prognostic algorithms for estimating remaining useful life of batteries. Transactions of the Institute of Measurement and Control, 31(3-4):293-308, 2009.

[54] H.Huang, Hai.Wang, Y. Li, L.Zhang, and Z. Liu. Support vector machine based estimation of remaining useful life: current research status and future trends. Journal of Mechanical Science and Technology, 29(1):151-163, 2015.

[55] J.F. Hu and P. Tse. A Relevance Vector Machine-Based Approach with Application to Oil Sand Pump Prognostics. Sensors, 13(9):12663-12686, 2013.

[56] P. J. Garcia Nieto, E. Garcia-Gonzalo, F. S. Lasheras, and F. J. Juez. Hybrid PSO-SVMbased method for forecasting of the remaining useful life for aircraft engines and evaluation of its reliability. Reliability Engineering and System Safety, 138:219-231, 2015.

[57] X. Li, J. Qian, and G.G. Wang. Fault prognostic based on hybrid method of state judgment and regression. Advances in Mechanical Engineering, 2013, 2013.

[58] L. Liao and F. K“ottig. Review of hybrid prognostics approaches for remaining useful life prediction of engineered systems, and an application to battery life prediction. IEEE Transactions on Reliability, 63(1):191-207, 2014.

[59] T. Wang, J. Yu, D. Siegel, and J. Lee. A similarity-based prognostic approach for Remaining Useful Life estimation of engineered systems. In Prognostics and Health Management, 2008. PHM 2008. International Conference on, pages 1-6, 2008.

[60] F. Xue, P. Bonissone, A. Varma, W. Yan, N. Eklund, and K. Goebel. An instance-based method for remaining useful life estimation for aircraft engines. Journal of Failure Analysis and Prevention, 8(2):199-206, 2008.

[61] R.A. Baurle and R.L. Gaffney. Extraction of One-Dimensional Flow Properties from Multidimensional Data Sets. Journal of Propulsion and Power, 24(4):704-714, 2008.

[62] S. Malinowski, B. Chebel-Morello, and N.Zerhouni. Remaining useful life estimation 
based on discriminating shapelet extraction. Reliability Engineering \& System Safety, 142:279-288, 2015.

[63] E. Ramasso and R. Gouriveau. Remaining Useful Lif Estimation by Classification of Predictions Based on a Neuro-Fuzzy System and Theory of Belief Functions. IEEE Transactions on Reliability, 63(2):555-566, 2014.

[64] Q. Zhang, P.W. Tse, X. Wan, and G. Xu. Remaining useful life estimation for mechanical systems based on similarity of phase space trajectory. Expert Systems with Applications, 42(5):2353- 2360, 2015.

[65] A. Mosallam, K. Medjaher, and N. Zerhouni. Data-driven prognostic method based on Bayesian approaches for direct remaining useful life prediction. Journal of Intelligent Manufacturing, 2014.

[66] E. Ramasso, M. Rombaut, and N. Zerhouni. Joint prediction of continuous and discrete states in time-series based on belief functions. IEEE Transactions on Cybernetics, 43(1):37-50, 2013.

[67] P. Wang, B.D. Youn, and C. Hu. A generic probabilistic framework for structural health prognostics and uncertainty management. Mechanical Systems and Signal Processing, 28:622-637, 2012.

[68] C. Hu, B.D. Youn, P. Wang, and J.T. Yoon. Ensemble of data-driven prognostic algorithms for robust prediction of remaining useful life. Reliability Engineering and System Safety, 103:120-135, 2012.

[69] M. Daigle, A. Bregon, and I. Roychoudhury. A Distributed Approach to System-Level Prognostics. In Annual conference of the Prognostics and Health management Society 2012, pages 71-82, 2012.

[70] R. Ahmad, S. Kamaruddin, I.A. Azid, and I.P. Almanar. Failure analysis of machinery component by considering external factors and multiple failure modes - A case study in the processing industry. Engineering Failure Analysis, 25:182-192, 2012.

[71] W. Huang and R.G. Askin. Reliability analysis of electronic devices with multiple competing failure modes involving performance aging degradation. Quality and Reliability Engineering International, 19(3):241-254, 2003.

[72] B.J. Bichon, J.M. McFarland, and S. Mahadevan. Efficient surrogate models for reliability analysis of systems with multiple failure modes. Reliability Engineering and System Safety, 96(10):1386-1395, 2011. 